\title{
Article \\ Existence and Uniqueness of Solutions to a Nabla Fractional Difference Equation with Dual Nonlocal Boundary Conditions
}

\author{
Nandhihalli Srinivas Gopal and Jagan Mohan Jonnalagadda * \\ Department of Mathematics, Birla Institute of Technology and Science Pilani, Hyderabad 500078, India; \\ p20190036@hyderabad.bits-pilani.ac.in \\ * Correspondence: jjaganmohan@hyderabad.bits-pilani.ac.in
}

check for

updates

Citation: Gopal, N.S.; Jonnalagadda,

J.M. Existence and Uniqueness of

Solutions to a Nabla Fractional

Difference Equation with Dual

Nonlocal Boundary Conditions.

Foundations 2022, 2, 151-166.

https://doi.org/10.3390/

foundations 2010009

Academic Editor: Sotiris K.

Ntouyas

Received: 30 December 2021

Accepted: 17 January 2022

Published: 21 January 2022

Publisher's Note: MDPI stays neutral with regard to jurisdictional claims in published maps and institutional affiliations.

Copyright: (C) 2022 by the authors. Licensee MDPI, Basel, Switzerland. This article is an open access article distributed under the terms and conditions of the Creative Commons Attribution (CC BY) license (https:// creativecommons.org/licenses/by/ $4.0 /)$.

\begin{abstract}
In this paper, we look at the two-point boundary value problem for a finite nabla fractional difference equation with dual non-local boundary conditions. First, we derive the associated Green's function and some of its properties. Using the Guo-Krasnoselkii fixed point theorem on a suitable cone and under appropriate conditions on the non-linear part of the difference equation, we establish sufficient requirements for at least one and at least two positive solutions of the boundary value problem. Next, we discuss the existence and uniqueness of solutions to the considered problem. For this purpose, we use Brouwer and Banach fixed point theorem, respectively. Finally, we provide a few examples to illustrate the applicability of established results.
\end{abstract}

Keywords: nabla fractional difference; boundary value problem; fixed point; positive solution; existence; uniqueness

\section{Introduction}

Over the last few decades, the theory of fractional calculus has been extensively developed due to its properties, generalizing most results of differential calculus and its non-local nature of fractional derivatives. The contributions of several mathematicians over the span of three centuries have resulted in a robust theory of fractional differential equations for real functions. Its roots can be traced back to the Leibniz letter dated "30 September 1695". Today fractional calculus has been successfully used for mathematical modelling in the fields of medical sciences, computational biology, economics, physics and several areas of engineering in the past three decades. For further applications and historical literature, we refer here to a few classical texts on fractional calculus by Miller and Ross [1], Samko et al. [2], Podlubny [3] and Kilbas et al. [4].

On the other side of the coin, nabla fractional calculus is a branch of mathematics, which deals with arbitrary order differences and sums in the backward sense. The theory of nabla fractional calculus is relatively young, with the most prominent works done in the past decade. The notion of nabla fractional difference and sum can be traced back to the work of Gray and Zhang [5], and Miller and Ross [6]. In this line, Atici and Eloe [7] developed nabla fractional Riemann-Liouville difference operator, initiated the study of nabla fractional initial value problem and established exponential law, product rule, and nabla Laplace transform. Following their works, the contributions of several mathematicians have made the theory of discrete fractional calculus a fruitful field of research in science and engineering, we refer here a few applications of discrete fractional equations [8-10]. We also refer here to a recent monograph by Goodrich and Peterson [11] and the references therein, which is an excellent source for all those who wish to work in this field.

The study of boundary value problems (BVPs) has a long past and can be followed back to the work of Euler and Taylor on vibrating strings. On the discrete fractional side, there is a sudden growth in interest for the development of nabla fractional BVPs. Many authors have studied nabla fractional BVPs recently. To name a few, Ahrendt [12], Goar [13], 
and Ikram [14] worked with self-adjoint Caputo nabla BVPs. Brackins [15] studied a particular class of self-adjoint Riemann-Liouville nabla BVPs and derived the Green's function associated with it along with a few of its properties. Gholami et al. [16] obtained the Green's function for a non-homogeneous Riemann-Liouville nabla BVP with Dirichlet boundary conditions. Jonnalagadda [17-22] analyzed some qualitative properties of twopoint non-linear Riemann-Liouville nabla BVPs associated with a variety of boundary conditions. Goodrich $[23,24]$ analyzed discrete fractional BVP's with non-local conditions in the delta case. To the best of our knowledge, there are no results for the solutions of nabla fractional BVP's with non-local conditions.

We consider the following boundary value problem with dual non-local conditions

$$
\left\{\begin{array}{l}
-\left(\nabla_{\rho(o)}^{\vartheta} v\right)(z)=q(z, v(z)), \quad z \in \mathbb{N}_{o+2}^{p} \\
v(o)=g_{1}(v), \quad v(p)=g_{2}(v),
\end{array}\right.
$$

where $o, p \in \mathbb{R}$ with $p-o \in \mathbb{N}_{3}, 1<\vartheta<2, q: \mathbb{N}_{o+2}^{p} \times \mathbb{R} \rightarrow \mathbb{R}$ and the functionals $g_{1}, g_{2}: \mathbb{R} \rightarrow \mathbb{R}$. The present paper is organized as follows: Section 2 contains a few preliminaries on discrete fractional calculus. In Section 3, we construct the Green's function corresponding to (1) and state a few of its properties. In Section 4, we study the existence of at least one and at least two positive solutions using Guo-Kranoselskii fixed point theorem on cones. In Section 5, we obtain sufficient conditions on the existence and uniqueness of solutions for the proposed class of boundary value problems using Brouwer and contraction mapping theorems, respectively. Finally, we conclude this article with a few examples.

\section{Preliminaries}

The set of all real numbers and positive integers are denoted by $\mathbb{R}$ and $\mathbb{Z}^{+}$, respectively. We use the following notations, definitions, and known results of nabla fractional calculus [11]. We assume empty sums and products are 0 and 1 , respectively.

Definition 1. For $o \in \mathbb{R}$, the sets $\mathbb{N}_{o}$ and $\mathbb{N}_{o}^{p}$, where $p-o \in \mathbb{Z}^{+}$, are defined by

$$
\mathbb{N}_{o}=\{o, o+1, o+2, \ldots\}, \quad \mathbb{N}_{o}^{p}=\{o, o+1, o+2, \ldots, p\} .
$$

Definition 2. We define the backward jump operator, $\rho: \mathbb{N}_{o+1} \longrightarrow \mathbb{N}_{o}$, by

$$
\rho(z)=z-1, \quad z \in \mathbb{N}_{o+1}
$$

Let $v: \mathbb{N}_{o} \rightarrow \mathbb{R}$ and $M \in \mathbb{N}_{1}$. The first order backward (nabla) difference of $v$ is defined by $(\nabla v)(z)=v(z)-v(z-1)$, for $z \in \mathbb{N}_{o+1}$, and the Mth-order nabla difference of $v$ is defined recursively by $\left(\nabla^{M_{v}}\right)(z)=\left(\nabla\left(\nabla^{M-1} v\right)\right)(z)$, for $z \in \mathbb{N}_{o+M}$.

Definition 3 (See [11]). Let $z \in \mathbb{R} \backslash\{\ldots,-2,-1,0\}$ and $r \in \mathbb{R}$ such that $(z+r) \in \mathbb{R} \backslash$ $\{\ldots,-2,-1,0\}$, the generalized rising function is defined by

$$
z^{\bar{r}}=\frac{\Gamma(z+r)}{\Gamma(z)} .
$$

Here $\Gamma(\cdot)$ denotes the Euler gamma function. Also, if $z \in\{\ldots,-2,-1,0\}$ and $r \in \mathbb{R}$ such that $(z+r) \in \mathbb{R} \backslash\{\ldots,-2,-1,0\}$, then we use the convention that $z^{\bar{r}}=0$.

Definition 4 (See [11]). Let $z, o \in \mathbb{R}$ and $\xi \in \mathbb{R} \backslash\{\ldots,-2,-1\}$. The $\xi$ th-order nabla fractional Taylor monomial is given by

$$
H_{\xi}(z, o)=\frac{(z-o)^{\bar{\xi}}}{\Gamma(\xi+1)^{\prime}}
$$

given that the right-hand side exists. 
We observe the following properties of the nabla fractional Taylor monomials.

Lemma 1 (See $[14,18])$. Let $\xi>-1$ and $t \in \mathbb{N}_{0}$. Then the following hold:

i. If $z \in \mathbb{N}_{\rho(t)}$, then $H_{\xi}(z, \rho(t)) \geq 0$ and if $z \in \mathbb{N}_{t}$, then $H_{\xi}(z, \rho(t))>0$.

ii. If $z \in \mathbb{N}_{t}$ and $-1<\xi<0$, then $H_{\xi}(z, \rho(t))$ is an increasing function of $t$.

iii. If $z \in \mathbb{N}_{t+1}$ and $-1<\xi<0$, then $H_{\xi}(z, \rho(t))$ is a decreasing function of $z$.

iv. If $z \in \mathbb{N}_{\rho(t)}$ and $\xi>0$, then $H_{\xi}(z, \rho(t))$ is a decreasing function of $t$.

v. If $z \in \mathbb{N}_{\rho(t)}$ and $\xi \geq 0$, then $H_{\xi}(z, \rho(t))$ is a non-decreasing function of $z$.

vi. If $z \in \mathbb{N}_{t}$ and $\xi>0$, then $H_{\xi}(z, \rho(t))$ is an increasing function of $z$.

vii. If $0<v \leq \xi$, then $H_{v}(z, o) \leq H_{\xi}(z, o)$, for each fixed $z \in \mathbb{N}_{o}$.

Lemma 2. Let $o$, $p$ be two real numbers such that $0<0 \leq p$ and $1<\vartheta<2$. Then $\frac{(o-t)^{\overline{\theta-1}}}{(p-t)^{\bar{\theta}-1}}$ is a decreasing function of $t$ for $t \in \mathbb{N}_{0}^{o-1}$.

Proof. It is enough to show that $\nabla\left(\frac{(o-t)^{\overline{\theta-1}}}{(p-t)^{\overline{\theta-1}}}\right)<0$.

Consider

$$
\begin{aligned}
& \nabla\left(\frac{(o-t)^{\overline{\vartheta-1}}}{(p-t)^{\overline{\vartheta-1}}}\right) \\
& =\frac{-(p-t)^{\overline{\vartheta-1}}(\vartheta-1)(o-\rho(t))^{\overline{\vartheta-2}}+(o-t)^{\overline{\vartheta-1}}(\vartheta-1)(p-\rho(t))^{\overline{\vartheta-2}}}{(p-t)^{\overline{\vartheta-1}}(p-\rho(t))^{\overline{\vartheta-1}}} \\
& =\frac{(\vartheta-1)\left(-(p-t)(p-\rho(t))^{\overline{\vartheta-2}}(o-\rho(t))^{\overline{\vartheta-2}}+(o-t)(o-\rho(t))^{\overline{\vartheta-2}}(p-\rho(t))^{\overline{\vartheta-2}}\right)}{(p-t)^{\overline{\vartheta-1}}(p-\rho(t))^{\overline{\vartheta-1}}} \\
& =\frac{(\vartheta-1)(p-\rho(t))^{\overline{\vartheta-2}}(o-\rho(t))^{\overline{\vartheta-2}}(-p+t+o-t)}{(p-t)^{\overline{\vartheta-1}}(p-\rho(t))^{\overline{\vartheta-1}}} \\
& =\frac{(\vartheta-1)(p-\rho(t))^{\overline{\vartheta-2}}(o-\rho(t))^{\overline{\vartheta-2}}(o-p)}{(p-t)^{\overline{\vartheta-1}}(p-\rho(t))^{\overline{\vartheta-1}}} .
\end{aligned}
$$

Since $p>0$, it follows from Lemma 1 that $\nabla\left(\frac{(o-t)^{\overline{\theta-1}}}{(p-t)^{\overline{\theta-1}}}\right)<0$. The proof is complete.

Definition 5 (See [11]). Let $v: \mathbb{N}_{o+1} \rightarrow \mathbb{R}$ and $v>0$. The $v$ th-order nabla sum of $v$ is given by

$$
\left(\nabla_{o}^{-v} v\right)(z)=\sum_{t=o+1}^{z} H_{v-1}(z, \rho(t)) v(t), \quad z \in \mathbb{N}_{o+1} .
$$

Definition 6 (See [11]). Let $v: \mathbb{N}_{o+1} \rightarrow \mathbb{R}, v>0$ and choose $A \in \mathbb{N}_{1}$ such that $A-1<v \leq A$. The vth-order Riemann-Liouville nabla difference of $v$ is given by

$$
\left(\nabla_{o}^{v} v\right)(z)=\left(\nabla^{A}\left(\nabla_{o}^{-(A-v)} v\right)\right)(z), \quad z \in \mathbb{N}_{o+A}
$$

Theorem 1 (See [11]). Assume $\mu>0$ and $A-1<\mu \leq A$. Then a general solution of $\nabla_{o}^{\mu} v(z)=0$ is given by

$$
v(z)=c_{1} H_{\mu-1}(z, o)+c_{2} H_{\mu-2}(z, o)+\cdots+c_{A} H_{\mu-A}(z, o), \quad \text { for } \quad z \in \mathbb{N}_{o} .
$$




\section{Green's Function}

In this section, we will build the Green's function formula for our boundary value problem (1) and derive a few properties from it that will be used throughout the rest of the paper.

Theorem 2 (See [15]). The following nabla fractional boundary value problem

$$
\begin{aligned}
-\left(\nabla_{\rho(o)}^{\vartheta} v\right)(z) & =h(z), \quad z \in \mathbb{N}_{o+2}^{p}, \\
v(o) & =v(p)=0,
\end{aligned}
$$

where $0, p \in \mathbb{R}$ with $p-o \in \mathbb{N}_{3}, 1<\vartheta<2$ and $h: \mathbb{N}_{o+2}^{p} \rightarrow \mathbb{R}$. Then (2) has the unique solution

$$
v(z)=\sum_{t=o+2}^{p} G(z, t) h(t), \quad z \in \mathbb{N}_{o}^{p}
$$

where Green's function $G(z, t)$ is given by

$$
G(z, t)= \begin{cases}G_{1}(z, t)=\frac{H_{\vartheta-1}(z, o)}{H_{\vartheta-1}(p, o)} H_{\vartheta-1}(p, \rho(t)), & z \in \mathbb{N}_{o}^{t-1}, \\ G_{2}(z, t)=\frac{H_{\vartheta-1}(z, o)}{H_{\vartheta-1}(p, o)} H_{\vartheta-1}(p, \rho(t))-H_{\vartheta-1}(z, \rho(t)), & z \in \mathbb{N}_{t}^{p} .\end{cases}
$$

Lemma 3. The equivalent form of the following homogeneous nabla fractional boundary value problem with non-local conditions

$$
\left\{\begin{array}{l}
-\left(\nabla_{\rho(o)}^{\vartheta} w\right)(z)=0, \quad \text { for } \quad z \in \mathbb{N}_{o+2^{\prime}}^{p} \\
w(o)=g_{1}(w), \quad w(p)=g_{2}(w)
\end{array}\right.
$$

is given by

$$
w(z)=g_{1}(w)\left(\frac{p-z}{p-o}\right) \frac{(z-o+1)^{\overline{\vartheta-2}}}{\Gamma(\vartheta-1)}+g_{2}(w) \frac{(z-o)^{\overline{\vartheta-1}}}{(p-o)^{\overline{\vartheta-1}}}, \quad z \in \mathbb{N}_{o}^{p}
$$

Proof. From Theorem 1, the general solution of the equation $-\left(\nabla_{\rho(o)}^{\vartheta} w\right)(z)=0$, is given by

$$
w(z)=c_{1}(z-o+1)^{\overline{\vartheta-1}}+c_{2}(z-o+1)^{\overline{\vartheta-2}}, \quad z \in \mathbb{N}_{o}^{p}
$$

where $c_{1}$ and $c_{2}$ are arbitrary constants. Using $w(o)=g_{1}(w)$ and $w(p)=g_{2}(w)$, respectively in (7), we have

$$
\begin{aligned}
\frac{g_{1}(w)}{\Gamma(\vartheta-1)} & =c_{1}(\vartheta-1)+c_{2}, \\
g_{2}(w) & =c_{1}(p-o+1)^{\overline{\vartheta-1}}+c_{2}(p-o+1)^{\overline{\vartheta-2}} .
\end{aligned}
$$

Now, solving the above system of equations for $c_{1}$ and $c_{2}$, we have

$$
\begin{aligned}
& c_{1}=-\frac{g_{1}(w)(p-o+1)^{\overline{\vartheta-2}}}{\Gamma(\vartheta-1)(p-o)^{\overline{\vartheta-1}}}+\frac{g_{2}(w)}{(p-o)^{\overline{\vartheta-1}}} \\
& c_{2}=\frac{g_{1}(w)}{\Gamma(\vartheta-1)}-(\vartheta-1)\left[-\frac{g_{1}(w)(p-o+1)^{\overline{\vartheta-2}}}{\Gamma(\vartheta-1)(p-o)^{\overline{\vartheta-1}}}+\frac{g_{2}(w)}{(p-o)^{\overline{\vartheta-1}}}\right] .
\end{aligned}
$$


Substituting $c_{1}$ and $c_{2}$ in (7), we have

$$
\begin{aligned}
w(z) & =\left[\frac{g_{2}(w)}{(p-o)^{\bar{\vartheta}-1}}-\frac{g_{1}(w)(p-o+1)^{\overline{\vartheta-2}}}{\Gamma(\vartheta-1)(p-o)^{\overline{\vartheta-1}}}\right](z-o+1)^{\overline{\vartheta-1}} \\
& +\left[\frac{g_{1}(w)}{\Gamma(\vartheta-1)}-(\vartheta-1)\left[-\frac{g_{1}(w)(p-o+1)^{\overline{\vartheta-2}}}{\Gamma(\vartheta-1)(p-o)^{\bar{\vartheta}-1}}+\frac{g_{2}(w)}{(p-o)^{\overline{\vartheta-1}}}\right]\right](z-o+1)^{\overline{\vartheta-2}} \\
& =\frac{g_{1}(w)(p-o+1) \bar{\vartheta}}{\Gamma(\vartheta-1)(p-o)^{\bar{\vartheta}-1}}\left[(\vartheta-1)(z-o+1)^{\overline{\vartheta-2}}-(z-o+1)^{\overline{\vartheta-1}}\right] \\
& +\frac{g_{2}(w)}{(p-o)^{\overline{\vartheta-1}}}\left[(z-o+1)^{\overline{\vartheta-1}}-(\vartheta-1)(z-o+1)^{\overline{\vartheta-2}}\right]+\frac{g_{1}(w)}{\Gamma(\vartheta-1)}(z-o+1)^{\overline{\vartheta-2}} \\
& =\frac{g_{1}(w)}{\Gamma(\vartheta-1)}\left[(z-o+1)^{\overline{\vartheta-2}}-\frac{(p-o+1)^{\overline{\vartheta-2}}(z-o)^{\overline{\vartheta-1}}}{(p-o)^{\vartheta-1}}\right]+g_{2}(w) \frac{(z-o)^{\overline{\vartheta-1}}}{(p-o)^{\overline{\vartheta-1}}} \\
& =g_{1}(w)\left(\frac{p-z}{p-o}\right) \frac{(z-o+1)^{\overline{\vartheta-2}}}{\Gamma(\vartheta-1)}+g_{2}(w) \frac{(z-o)^{\overline{\vartheta-1}}}{(p-o)^{\overline{\vartheta-1}}} .
\end{aligned}
$$

The proof is complete.

Lemma 4. $w$ satisfies the following property:

$$
\max _{z \in \mathbb{N}_{o}^{p}} w(z) \leq g_{1}(w)+g_{2}(w)
$$

where $w$ is given by (7).

\section{Proof. Consider}

$$
w(z)=\left(\frac{p-z}{p-o}\right) \frac{(z-o+1)^{\overline{\vartheta-2}}}{\Gamma(\vartheta-1)} g_{1}(w)+\frac{(z-o)^{\overline{\vartheta-1}}}{(p-o)^{\overline{\vartheta-1}}} g_{2}(w) .
$$

Clearly, function $\left(\frac{p-z}{p-o}\right)$ is decreasing with respect to $z$ for $z \in \mathbb{N}_{o}^{p}$. It follows from Lemma 1 that function $\frac{(z-o+1)^{\overline{\theta-2}}}{\Gamma(\vartheta-1)}$ is decreasing with respect to $z$ and $\frac{(z-o)^{\frac{\theta-1}{(p-o}}}{(p-1}$ is an increasing function of $z$ for $z \in \mathbb{N}_{o}^{p}$. Thus, we have

$$
\begin{gathered}
\max _{z \in \mathbb{N}_{o}^{p}}\left(\frac{p-z}{p-o}\right)=1, \\
\max _{z \in \mathbb{N}_{o}^{p}} \frac{(z-o+1)^{\overline{\vartheta-2}}}{\Gamma(\vartheta-1)}=\frac{(o-o+1)^{\overline{\vartheta-2}}}{\Gamma(\vartheta-1)}=1, \\
\max _{z \in \mathbb{N}_{o}^{p}} \frac{(z-o)^{\overline{\vartheta-1}}}{(p-o)^{\overline{\vartheta-1}}}=1,
\end{gathered}
$$

implying that

$$
w(z) \leq g_{1}(w)+g_{2}(w), \quad \text { for } z \in \mathbb{N}_{o}^{p} .
$$

The proof is complete. 
Theorem 3 (See [17]). Let $1<\vartheta<2$ and $q: \mathbb{N}_{o}^{p} \times \mathbb{R} \rightarrow \mathbb{R}$. The equivalent form of (1) is given by

$$
v(z)=A(z) g_{1}(v)+B(z) g_{2}(v)+\sum_{t=o+2}^{p} G(z, t) q(t, v(t)), \quad z \in \mathbb{N}_{o}^{p}
$$

where $A(z)=\left(\frac{p-z}{p-o}\right) \frac{(z-o+1)^{\overline{\vartheta-2}}}{\Gamma(\vartheta-1)}, B(z)=\frac{(z-o)^{\overline{\vartheta-1}}}{(p-o)^{\overline{\vartheta-1}}}$ and Green's function $G(z, t)$ is given by (4), respectively.

Theorem 4 (See $[15,17])$. The Green's function $G(z, t)$ defined in (4) satisfies the following properties:

$$
\begin{array}{ll}
\text { i. } & G(o, t)=G(p, t)=0, \text { for all } s \in \mathbb{N}_{o+1}^{p} . \\
\text { ii. } & G(z, o+1)=0, \text { for all } z \in \mathbb{N}_{o}^{p} . \\
\text { iii. } & G(z, t)>0, \text { for all }(z, t) \in \mathbb{N}_{o+1}^{p-1} \times \mathbb{N}_{o+2}^{p} . \\
\text { iv. } & \max _{z \in \mathbb{N}_{o+1}^{p-1}} G(z, t)=G(t-1, t) \text { for all } t \in \mathbb{N}_{o+2}^{p} . \\
\text { v. } & \sum_{t=o+1}^{p} G(z, t) \leq \Lambda, \text { for all }(z, t) \in \mathbb{N}_{o}^{p} \times \mathbb{N}_{o+1}^{p} \text {, where } \\
& \Lambda=\left(\frac{p-o-1}{\vartheta \Gamma(\vartheta+1)}\right)\left(\frac{(\vartheta-1)(p-o)+1}{\vartheta}\right)^{\overline{\vartheta-1}} .
\end{array}
$$

\section{Existence of Positive Solution}

In this section, we show the existence of at least one positive solution for the boundary value problem (1), using Guo-Krasnoselskii fixed point theorem on a suitable cone.

Definition 7. Let $\mathcal{B}$ be a Banach space over $\mathbb{R}$. A closed nonempty subset $\mathcal{C}$ of $\mathcal{B}$ is said to be a cone provided,

(i) $e x+f y \in \mathcal{C}$, for all $x, y \in \mathcal{C}$ and all $e, f \geq 0$, and

(ii) $x \in \mathcal{C}$ and $-x \in \mathcal{C}$ implies $x=0$.

Definition 8. An operator is called completely continuous if it is continuous and maps bounded sets into pre-compact sets.

Lemma 5 (See [25]). [Guo-Krasnoselskii fixed point theorem] Let $\mathcal{B}$ be a Banach space and $\mathcal{C} \subseteq \mathcal{B}$ be a cone. Assume that $\Omega_{1}$ and $\Omega_{2}$ are open sets contained in $\mathcal{B}$ such that $0 \in \Omega_{1}$ and $\bar{\Omega}_{1} \subseteq \Omega_{2}$. Assume further that $T: \mathcal{C} \cap\left(\bar{\Omega}_{2} \backslash \Omega_{1}\right) \longrightarrow \mathcal{C}$ is a completely continuous operator. If either

i. $\quad\|T v\| \leq\|v\|$ for $v \in \mathcal{C} \cap \partial \Omega_{1}$ and $\|T v\| \geq\|v\|$ for $v \in \mathcal{C} \cap \partial \Omega_{2} ;$ or

ii. $\quad\|T v\| \geq\|v\|$ for $v \in \mathcal{C} \cap \partial \Omega_{1}$ and $\|T v\| \leq\|v\|$ for $v \in \mathcal{C} \cap \partial \Omega_{2}$;

holds, then $T$ has at least one fixed point in $\mathcal{C} \cap\left(\bar{\Omega}_{2} \backslash \Omega_{1}\right)$.

We establish the following lemmas, which will be used later in the proof of our main result.

Lemma 6. There exits a number $\lambda \in(0,1)$, such that

$$
\min _{z \in \mathbb{N}_{c}^{d}} G(z, t) \geq \lambda \max _{z \in \mathbb{N}_{o}^{p}} G(z, t)=\lambda G(t-1, t)
$$

where, $c, d \in \mathbb{N}_{o+1}^{p-1}, c=o+\left\lceil\frac{p-o+1}{4}\right\rceil$ and $d=o+3\left\lfloor\frac{p-o+1}{4}\right\rfloor$.

Proof. By using the properties of Green's function and Taylor monomial from Definition 4, Lemma 1 and Theorem 4, respectively. 
Consider, for $t \in \mathbb{N}_{o+2}^{p}$

$$
\frac{G(z, t)}{G(t-1, t)}= \begin{cases}\frac{(z-o)^{\overline{\theta-1}}}{(t-o-1)^{\bar{\theta}-1}} & \text { for } t>z, \\ \frac{(z-o)^{\overline{\theta-1}}}{(t-o-1)^{\bar{\theta}-1}}-\frac{(z-t+1)^{\overline{\theta-1}}(p-o)^{\overline{\theta-1}}}{(p-t+1)^{\bar{\theta}-1}(t-o+1)^{\bar{\theta}-1}}, & \text { for } t \leq z .\end{cases}
$$

Now, for $t>z$ and $c \leq z \leq d$. Function $G_{1}(z, t)$ is increasing with respect to $z$. Then,

$$
\begin{aligned}
\min _{z \in \mathbb{N}_{c}^{d}} G_{1}(z, t) & =G_{1}(c, t) \\
& =\frac{(c-o)^{\overline{\vartheta-1}}}{(p-o)^{\overline{\vartheta-1}}}(p-t+1)^{\overline{\vartheta-1}} .
\end{aligned}
$$

For $z \geq t$ and $c \leq z \leq d$, function $G_{2}(z, t)$ is decreasing with respect to $z$. Then,

$$
\begin{aligned}
\min _{z \in \mathbb{N}_{c}^{d}} G_{2}(z, t) & =G_{2}(d, t), \\
& =\frac{(d-o)^{\overline{\vartheta-1}}}{(p-o)^{\overline{\vartheta-1}}}(p-t+1)^{\overline{\vartheta-1}}-(d-t+1)^{\overline{\vartheta-1}} .
\end{aligned}
$$

Thus,

$$
\begin{aligned}
\min _{z \in \mathbb{N}_{c}^{d}} G(z, t) & = \begin{cases}G_{2}(d, t), & \text { for } t \in \mathbb{N}_{o+2}^{c}, \\
\min \left(G_{2}(d, t), G_{2}(c, t)\right), & \text { for } t \in \mathbb{N}_{c+1}^{d-1} \\
G_{1}(c, t), & \text { for } t \in \mathbb{N}_{d^{\prime}}^{p}\end{cases} \\
& = \begin{cases}G_{2}(d, t), & \text { for } t \in \mathbb{N}_{o+2^{r}}^{r} \\
G_{1}(c, t), & \text { for } t \in \mathbb{N}_{r}^{p},\end{cases}
\end{aligned}
$$

where $c<r<d$. Consider

$$
\frac{\min _{z \in \mathbb{N}_{c}^{d}} G(z, t)}{G(t-1, t)}= \begin{cases}\frac{(d-o)^{\overline{\theta-1}}}{(t-o+1)^{\bar{\theta}-1}}-\frac{(d-t+1)^{\overline{\theta-1}}(p-o)^{\overline{\theta-1}}}{(p-t+1)^{\vartheta-1}(t-o+1)^{\bar{\theta}-1}}, & \text { for } t \in \mathbb{N}_{o+2}^{r} \\ \frac{(c-o)^{\vartheta-1}}{(t-o+1)^{\bar{\theta}-1}}, & \text { for } t \in \mathbb{N}_{r}^{p} .\end{cases}
$$

Thus,

$$
\min _{z \in \mathbb{N}_{c}^{d}} G(z, t) \geq \lambda(t) \max _{z \in \mathbb{N}_{o}^{p}} G(z, t)
$$

where

$$
\lambda(t)=\min \left[\frac{(c-o)^{\overline{\vartheta-1}}}{(t-o+1)^{\overline{\vartheta-1}}}, \frac{(d-o)^{\overline{\vartheta-1}}}{(t-o+1)^{\overline{\vartheta-1}}}-\frac{(d-t+1)^{\overline{\vartheta-1}}(p-o)^{\overline{\vartheta-1}}}{(p-t+1)^{\overline{\vartheta-1}}(t-o+1)^{\overline{\vartheta-1}}}\right] .
$$

Let for $t \in \mathbb{N}_{r}^{p}$, denote

$$
\begin{aligned}
\lambda_{1}(t) & =\frac{(c-o)^{\overline{\vartheta-1}}}{(t-o+1)^{\overline{\vartheta-1}}} \\
& \geq \frac{(c-o)^{\overline{\vartheta-1}}}{(p-o+1)^{\overline{\vartheta-1}}} .
\end{aligned}
$$


Similarly, for $t \in \mathbb{N}_{o+2}^{r}$, we take

$$
\lambda_{2}(t)=\frac{1}{(t-o+1)^{\overline{\vartheta-1}}}\left[(d-o)^{\overline{\vartheta-1}}-\frac{(d-t+1)^{\overline{\vartheta-1}}(p-o)^{\overline{\vartheta-1}}}{(p-t+1)^{\overline{\vartheta-1}}}\right] .
$$

By Lemmas 1 and 2, we see that function $\frac{(d-t+1)^{\overline{\vartheta-1}}}{(p-t+1)^{\bar{\vartheta}-1}}$ is decreasing for $t \in \mathbb{N}_{o+2}^{r}$. Then

$$
\begin{aligned}
\lambda_{2}(t) & \geq \frac{1}{(t-o+1)^{\overline{\vartheta-1}}}\left[(d-o)^{\overline{\vartheta-1}}-\frac{(d-o-1)^{\overline{\vartheta-1}}(p-o)^{\overline{\vartheta-1}}}{(p-o-1)^{\overline{\vartheta-1}}}\right] \\
& \geq \frac{1}{(d-o)^{\overline{\vartheta-1}}}\left[(d-o)^{\overline{\vartheta-1}}-\frac{(d-o-1)^{\overline{\vartheta-1}}(p-o)^{\overline{\vartheta-1}}}{(p-o-1)^{\overline{\vartheta-1}}}\right] .
\end{aligned}
$$

Thus,

$$
\min _{z \in \mathbb{N}_{c}^{d}} G(z, t) \geq \lambda \max _{z \in \mathbb{N}_{o}^{p}} G(z, t)
$$

where

$$
\lambda=\min \left[\frac{(c-o)^{\overline{\vartheta-1}}}{(p-o+1)^{\overline{\vartheta-1}}}, 1-\frac{(d-o-1)^{\overline{\vartheta-1}}(p-o)^{\overline{\vartheta-1}}}{(p-o-1)^{\overline{\vartheta-1}}(d-o)^{\overline{\vartheta-1}}}\right] .
$$

Since $G_{1}(c, t)>0$ and $G_{2}(d, t)>0$, we have $\lambda(t)>0$ for all $t \in \mathbb{N}_{o+2}^{p}$, implying $\lambda>0$. It would be suffice to prove that one of the terms

$$
\frac{(c-o)^{\overline{\vartheta-1}}}{(p-o+1)^{\overline{\vartheta-1}}}, 1-\frac{(d-o-1)^{\overline{\vartheta-1}}(p-o)^{\overline{\vartheta-1}}}{(p-o-1)^{\overline{\vartheta-1}}(d-o)^{\overline{\vartheta-1}}}<1 .
$$

It follows from Lemma 1 that

$$
\frac{(c-o)^{\overline{\vartheta-1}}}{(p-o+1)^{\overline{\vartheta-1}}}<1
$$

Therefore, we conclude that $\lambda \in(0,1)$. The proof is complete.

Lemma 7. There exists a number $\lambda_{0} \in(0,1)$ such that

$$
\min _{z \in \mathbb{N}_{c}^{d}} w(z) \geq \lambda_{0}\left(\max _{z \in \mathbb{N}_{o}^{p}} A(z) g_{1}(w)+\max _{z \in \mathbb{N}_{o}^{p}} B(z) g_{2}(w)\right) .
$$

where $w$ is given by (6).

Proof. Clearly, function $\left(\frac{p-z}{p-o}\right)$ is decreasing with respect to $z$ for $z \in \mathbb{N}_{o}^{p}$. It follows from Lemma 1 that $\frac{(z-o+1)^{\overline{\vartheta-2}}}{\Gamma(\vartheta-1)}$ is a decreasing function of $z$ and function $\frac{(z-o)^{\overline{\vartheta-1}}}{(p-o)^{\bar{\vartheta}-1}}$ is an increasing with respect to $z$ for $z \in \mathbb{N}_{o}^{p}$. Then, there exists $M_{1}, M_{2}>0$ such that

$$
\begin{aligned}
& \min _{z \in \mathbb{N}_{c}^{d}}\left(\frac{p-z}{p-o}\right) \frac{(z-o+1)^{\overline{\vartheta-2}}}{\Gamma(\vartheta-1)}=M_{1}, \\
& \min _{z \in \mathbb{N}_{c}^{d}} \frac{(z-o)^{\overline{\vartheta-1}}}{(p-o)^{\overline{\vartheta-1}}}=M_{2}<1 .
\end{aligned}
$$


Take $\lambda_{0}=\min \left(M_{1}, M_{2}\right)$. Clearly $\lambda_{0} \in(0,1)$. Thus, for all $z \in \mathbb{N}_{c}^{d}$, we have

$$
\begin{aligned}
w(z) & \geq \min _{z \in \mathbb{N}_{c}^{d}}\left[\left(\frac{p-z}{p-o}\right) \frac{(z-o+1)^{\overline{\vartheta-2}}}{\Gamma(\vartheta-1)}\right] g_{1}(w)+\min _{z \in \mathbb{N}_{c}^{d}}\left[\frac{(z-o)^{\overline{\vartheta-1}}}{(p-o)^{\bar{\vartheta}-1}}\right] g_{2}(w) \\
& =M_{1} g_{1}(w)+M_{2} g_{2}(w) \\
& \geq \lambda_{0} g_{1}(w)+\lambda_{0} g_{2}(w) \\
& =\lambda_{0}\left[\max _{z \in \mathbb{N}_{o}^{p}}\left(\frac{p-z}{p-o}\right) \frac{(z-o+1)^{\overline{\vartheta-2}}}{\Gamma(\vartheta-1)}\right] g_{1}(w)+\lambda_{0}\left[\max _{z \in \mathbb{N}_{o}^{p}} \frac{(z-o)^{\overline{\vartheta-1}}}{(p-o)^{\overline{\vartheta-1}}}\right] g_{2}(w) \\
& =\lambda_{0}\left(\max _{z \in \mathbb{N}_{o}^{p}} A(z) g_{1}(w)+\max _{z \in \mathbb{N}_{o}^{p}} B(z) g_{2}(w)\right) .
\end{aligned}
$$

The proof is complete.

Lemma 8. If $g_{1}, g_{2}$ are non-negative, then there exists a constant $\bar{\lambda} \in(0,1)$, such that

$$
\begin{aligned}
\min _{z \in \mathbb{N}_{c}^{d}} & \sum_{t=o+2}^{p} G(z, t) q(z, v(t))+\min _{z \in \mathbb{N}_{c}^{d}}\left(A(z) g_{1}(v)+B(z) g_{2}(v)\right) \\
& \geq \bar{\lambda} \max _{z \in \mathbb{N}_{o}^{p}}\left(\sum_{s=o+2}^{p} G(z, t) q(z, v(t))+A(z) g_{1}(v)+B(z) g_{2}(v)\right) .
\end{aligned}
$$

Proof. It follows from Lemmas 6 and 7 have

$$
\begin{aligned}
\min _{z \in \mathbb{N}_{c}^{d}} \sum_{t=o+2}^{p} G(z, t) f(t, v(t))+\min _{z \in \mathbb{N}_{c}^{d}}\left(A(z) g_{1}(v)+B(z) g_{2}(v)\right) \\
\quad \geq \sum_{t=o+2}^{p} \min _{z \in \mathbb{N}_{c}^{d}} G(z, t) q(t, v(t))+\lambda_{0}\left(\max _{z \in \mathbb{N}_{o}^{p}} A(z) g_{1}(v)+\max _{z \in \mathbb{N}_{o}^{p}} B(z) g_{2}(v)\right) \\
\geq \lambda \sum_{t=o+2}^{p} \max _{z \in \mathbb{N}_{o}^{p}} G(z, t) q(t, v(t))+\lambda_{0} \max _{z \in \mathbb{N}_{o}^{p}}\left(A(z) g_{1}(v)+B(z) g_{2}(v)\right) \\
\geq \bar{\lambda}\left(\max _{z \in \mathbb{N}_{o}^{p}} \sum_{t=o+2}^{p} G(z, t) f(t, v(t))+A(z) g_{1}(v)+B(z) g_{2}(v)\right) .
\end{aligned}
$$

where $\bar{\lambda}=\min \left(\lambda, \lambda_{0}\right) \in(0,1)$. The proof is complete.

We observe by Theorem 3 that the equivalent form of (1) is given by

$$
v(z)=A(z) g_{1}(v)+B(z) g_{2}(v)+\sum_{t=o+2}^{p} G(z, t) q(t, v(t)), \quad z \in \mathbb{N}_{o}^{p}
$$

where $A(z)=\left(\frac{p-z}{p-o}\right) \frac{(z-o+1)^{\overline{\vartheta-2}}}{\Gamma(\vartheta-1)}, B(z)=\frac{(z-o)^{\overline{\vartheta-1}}}{(p-o)^{\overline{\vartheta-1}}}$ and Green's function $G(z, t)$ is given by (4), respectively. 
Note that any solution $v: \mathbb{N}_{o}^{p} \rightarrow \mathbb{R}$ of (1) can be viewed as a real $(p-o+1)$-tuple vector. Consequently, $v \in \mathbb{R}^{p-o+1}$. Define the operator $T: \mathbb{R}^{p-o+1} \rightarrow \mathbb{R}^{p-o+1}$ by

$$
(T v)(z)=A(z) g_{1}(v)+B(z) g_{2}(v)+\sum_{t=o+2}^{p} G(z, t) q(t, v(t)), \quad z \in \mathbb{N}_{o}^{p} .
$$

Clearly, $v$ is a fixed point of $T$ if and only if $v$ is a solution of (1). We use the fact that $\mathcal{B}=\mathbb{R}^{p-o+1}$ is a Banach space equipped with the maximum norm

$$
\|v\|=\max _{z \in \mathbb{N}_{o}^{p}}|v(z)|,
$$

for any $v \in \mathcal{B}$. We define the cone $\mathcal{C}$ by

$$
\mathcal{C}=\left\{v \in \mathcal{B}: v(z) \geq 0 \text { and } \min _{z \in \mathbb{N}_{c}^{d}} v(z) \geq \bar{\lambda}\|v(z)\|\right\} .
$$

Note that summation operator $T$ is defined on a discrete finite set. Hence, operator $T$ is trivially completely continuous.

Take

$$
\eta=\frac{1}{\sum_{t=o+2}^{p} G(t-1, t)} .
$$

We state here the following hypothesis which will be used later

(H1) $\quad q(z, v) \geq 0,(z, v) \in \mathbb{N}_{o}^{p} \times[0, \infty)$ and $g_{1}(v), g_{2}(v) \geq 0, \forall v \in C\left[\mathbb{N}_{o+2}^{p} \rightarrow \mathbb{R}\right]$,

(H2) There exists a number $r>0$ such that $q(z, v) \leq \frac{r \eta}{2}$, whenever $0 \leq v \leq r$,

(H3) There exists a number $r>0$ such that $q(z, v) \geq \frac{r \eta}{\bar{\lambda}}$, whenever $\bar{\lambda} r \leq v \leq r$,

(H4) Assume that $\lim _{v \rightarrow 0^{+}} \min _{z \in \mathbb{N}_{o}^{p}} \frac{q(z, v)}{v}=\infty, \quad \lim _{v \rightarrow \infty} \min _{z \in \mathbb{N}_{o}^{p}} \frac{q(z, v)}{v}=\infty$,

(H5) Assume that $\lim _{v \rightarrow 0^{+}} \min _{z \in \mathbb{N}_{o}^{p}} \frac{q(z, v)}{v}=0, \quad \lim _{v \rightarrow \infty} \min _{z \in \mathbb{N}_{o}^{p}} \frac{q(z, v)}{v}=0$,

(G1) The functionals $g_{1}(v)$ and $g_{2}(v)$ are linear. In particular, we assume that

$$
g_{1}(v)=\sum_{i=o}^{p} m_{i} v(i) \text { and } g_{2}(v)=\sum_{i=o}^{p} n_{i} v(i),
$$

where $m_{i}, n_{i} \in \mathbb{R}$,

(G2) Assume $\sum_{i=o}^{p} m_{i}+\sum_{i=o}^{p} n_{i} \leq \frac{1}{2}$.

Lemma 9. Assume (H1) holds. Then, $T: \mathcal{C} \rightarrow \mathcal{C}$.

Proof. Let operator $T$ be as defined in (18), then by using Lemma 8, we have

$$
\begin{aligned}
\min _{z \in \mathbb{N}_{c}^{d}}(T v)(z) & \geq \min _{z \in \mathbb{N}_{c}^{d}} \sum_{t=o+2}^{p} G(z, t) q(t, v(t))+\min _{z \in \mathbb{N}_{c}^{d}}\left(A(z) g_{1}(v)+B(z) g_{2}(v)\right) \\
& \geq \bar{\lambda} \max _{z \in \mathbb{N}_{o}^{p}}\left(\sum_{t=o+2}^{p} G(z, t) q(z, v(t))+A(z) g_{1}(v)+B(z) g_{2}(v)\right) . \\
& \geq \bar{\lambda}\|T v\| .
\end{aligned}
$$

It is obvious that $(T v)(z) \geq 0$, whenever $v \in \mathcal{C}$ thus $T: \mathcal{C} \rightarrow \mathcal{C}$.

Theorem 5. Assume $q(z, v)$ satisfy conditions $\{(H 1),(H 2),(H 3)\}$. Also, $g_{1}(v), g_{2}(v)$ satisfy conditions $\{(H 1),(G 1),(G 2)\}$. Then, the boundary value problem (1) has at least one positive solution. 
Proof. We know that, $T: \mathcal{C} \rightarrow \mathcal{C}$ is completely continuous. For $0<r_{1}<r_{2}$, define the set $\Omega_{1}=\left\{v \in \mathcal{C}:\|v\|<r_{1}\right\}$. Clearly, $\Omega_{1} \subset \mathcal{B}$ is an open set with $0 \in \Omega_{1}$. Since $\|v\|=r_{1}$ for $v \in \partial \Omega_{1},(H 2),(G 1),(G 2)$ holds for all $v \in \partial \Omega_{1}$. Then we have for $v \in \mathcal{C} \cap \partial \Omega_{1}$, by using Lemma 4.

$$
\begin{aligned}
\|T v\| & \leq \max _{z \in \mathbb{N}_{p}^{o}} \sum_{t=o+2}^{p} G(z, t) q(t, v(t))+\max _{z \in \mathbb{N}_{o}^{p}} A(z) g_{1}(v)+\max _{z \in \mathbb{N}_{o}^{p}} B(z) g_{2}(v) \\
& \leq \sum_{t=o+2}^{p} \max _{z \in \mathbb{N}_{p}^{o}}[G(z, t)] q(t, v(t))+g_{1}(v)+g_{2}(v) \\
& \leq \frac{\eta r_{1}}{2} \sum_{t=o+2}^{p} G(t-1, t)+g_{1}(v)+g_{2}(v) \\
& \leq\left(\frac{1}{2}+\sum_{i=o}^{p} m_{i}+\sum_{i=o}^{p} n_{i}\right) r_{1} \leq r_{1} .
\end{aligned}
$$

Therefore $\|T v\| \leq\|v\|$, for $v \in \mathcal{C} \cap \partial \Omega_{1}$. Similarly, set $\Omega_{2}=\left\{v \in \mathcal{C}:\|v\|<r_{2}\right\}$. Clearly, $\Omega_{2} \in \beta$ is an open set and $\bar{\Omega}_{1} \subseteq \Omega_{2}$. Since $\|v\|=r_{2}$ for $v \in \partial \Omega_{2},(H 3),(G 1),(G 2)$ holds. We have

$$
\begin{aligned}
\|T v\| & \geq \min _{z \in \mathbb{N}_{c}^{d}}|T v(z)| \\
& \geq \min _{z \in \mathbb{N}_{c}^{d}} \sum_{t=o+2}^{p} G(z, t) q(t, v(t))+\min _{z \in \mathbb{N}_{c}^{d}} A(z) g_{1}(v)+\min _{z \in \mathbb{N}_{c}^{d}} B(z) g_{2}(v) \\
& \geq \bar{\lambda} \sum_{t=o+2}^{p} \max _{z \in \mathbb{N}_{p}^{o}}[G(z, t)] q(t, v(t)) \\
& \geq \bar{\lambda} \sum_{t=o+2}^{p} G(t-1, t) q(t, v(t)) \\
& \geq \bar{\lambda} \frac{r_{2} \eta}{\bar{\lambda}} \sum_{t=o+2}^{p} G(t-1, t)=r_{2} .
\end{aligned}
$$

Thus, we have $\|T v\| \geq\|v\|$, for $v \in \partial \Omega_{2} \cap \mathcal{C}$. By part (i) of Theorem 5, we conclude that operator $T$ has a fixed point $v_{0}$ in $\mathcal{C} \cap\left(\bar{\Omega}_{2} \backslash \Omega_{1}\right)$, satisfying $r_{1}<\left\|v_{0}\right\|<r_{2}$. The proof is complete.

Theorem 6. Assume $q(z, v)$ satisfy conditions $\{(H 1),(H 2),(H 4)\}$. Also, $g_{1}(v), g_{2}(v)$ satisfy conditions $\{(H 1),(G 1),(G 2)\}$. Then, the boundary value problem (1) has at least two positive solutions $v_{1}$ and $v_{2}$ with $0<\left\|v_{1}\right\|<r_{2}<\left\|v_{2}\right\|$.

Proof. Let us choose a number $N>0$ such that

$$
\frac{N \bar{\lambda}}{\eta}>1
$$

by condition (H4) there exists a number $r^{*}>0$ such that $r^{*}<r_{1}<r_{2}$ and $q(z, v) \geq N v$ for $v \in\left[0, r^{*}\right]$ and $z \in \mathbb{N}_{o}^{p}$. Define the set $\Omega_{r^{*}}=\left\{v \in \mathcal{C}:\|v\|<r^{*}\right\}$. Then we have 


$$
\begin{aligned}
\|T v\| & \geq \min _{z \in \mathbb{N}_{c}^{d}}|T v(z)| \\
& \geq \min _{z \in \mathbb{N}_{c}^{d}} \sum_{t=o+2}^{p} G(z, t) q(t, v(t))+\min _{z \in \mathbb{N}_{c}^{d}} A(z) g_{1}(v)+\min _{z \in \mathbb{N}_{c}^{d}} B(z) g_{2}(v) \\
& \geq \sum_{t=o+2}^{p} \min _{z \in \mathbb{N}_{c}^{d}}[G(z, t)] q(t, v(t)) \\
& \geq \bar{\lambda} \sum_{t=o+2}^{p} \max _{z \in \mathbb{N}_{p}^{o}}[G(z, t)] q(t, v(t)) \\
& \geq \bar{\lambda} N v \sum_{t=o+2}^{p} G(t-1, t)>r^{*} .
\end{aligned}
$$

Thus, we have $\|T v\|>\|v\|$, for $v \in \partial \Omega_{r^{*}} \cap \mathcal{C}$. Next for the same $N$, we can find a number $R_{1}>0$ such that $q(z, v) \geq N v$ for $v \geq R_{1}$ and $z \in \mathbb{N}_{o}^{p}$. Choose $R$ so that $R=\max \left\{r_{2}, \frac{R_{1}}{\bar{\lambda}}\right\}$. Define the set $\Omega_{R}=\{v \in \mathcal{C}:\|v\|<R\}$. We can show that $\|T v\| \geq\|v\|$, for $v \in \partial \Omega_{R} \cap \mathcal{C}$.

Finally define the set

$$
\Omega_{2}=\left\{v \in \mathcal{C}:\|v\|<r_{2}\right\} .
$$

Since $\|v\|=r_{2}$ condition (H2) holds for all $v \in \partial \Omega_{2}$. Then, we have

$$
\begin{aligned}
\|T v\| & \leq \max _{z \in \mathbb{N}_{p}^{o}} \sum_{t=o+2}^{p} G(z, t) q(t, v(t))+\max _{z \in \mathbb{N}_{o}^{p}} A(z) g_{1}(v)+\max _{z \in \mathbb{N}_{o}^{p}} B(z) g_{2}(v) \\
& \leq \sum_{t=o+2}^{p} \max _{z \in \mathbb{N}_{p}^{o}}[G(z, t)] q(t, v(t))+g_{1}(v)+g_{2}(v) \\
& \leq \frac{r_{2} \eta}{2} \sum_{t=o+2}^{p} G(t-1, t)+g_{1}(v)+g_{2}(v) \\
& \leq\left(\frac{1}{2}+\sum_{i=o}^{p} m_{i}+\sum_{i=o}^{p} n_{i}\right) r_{2} \leq r_{2} .
\end{aligned}
$$

Implying $\|T v\| \geq\|v\|$, for $v \in \partial \Omega_{r_{2}} \cap \mathcal{C}$. Hence, we conclude that $T$ has at least two fixed points say $v_{1} \in \Omega_{2} \backslash \hat{\Omega}_{r^{*}}$ and $v_{2} \in \Omega_{R} \backslash \hat{\Omega}_{2}$, where $\hat{\Omega}$ denoted the interior of the set $\Omega$. In particular (1) has at least two positive solutions, say $v_{1}$ and $v_{2}$ satisfying $0<\left\|v_{1}\right\|<r_{2}<\left\|v_{2}\right\|$. The proof is complete.

Theorem 7. Assume $q(z, v)$ satisfy conditions $\{(H 1),(H 3),(H 5)\}$. Also, $g_{1}(v), g_{2}(v)$ satisfy conditions $\{(H 1),(G 1),(G 2)\}$. Then, the boundary value problem (1) has at least two positive solutions $v_{1}$ and $v_{2}$ with $0<\left\|v_{1}\right\|<r_{2}<\left\|v_{2}\right\|$.

\section{Existence of Solutions}

In this section, we present the existence and uniqueness results of the boundary value problem (1) using Brouwer fixed point theorem and contraction mapping theorem, respectively and also, we construct an example to illustrate the same results.

Theorem 8 (See [25]). [Brouwer fixed point theorem]. Let $\mathcal{C}_{0}$ be a nonempty compact convex subset of $\mathbb{R}^{n}$ and $T$ be a continuous mapping of $\mathcal{C}_{0}$ into itself. Then, $T$ has a fixed point in $\mathcal{C}_{0}$.

Theorem 9. Assume $q(z, v), g_{1}(v)$ and $g_{2}(v)$ is continuous with respect to ' $v^{\prime}$, for each $z \in \mathbb{N}_{o}^{p}$. Assume there exist a positive constant $L$, such that

$$
\max _{-L \leq\|v\| \leq L}\left\{g_{1}(v), g_{2}(v)\right\} \leq \frac{L}{2(\Lambda+1)}
$$


and

$$
\max _{(z, v) \in \mathbb{N}_{o}^{p} \times[-L, L]}\{q(z, v)\} \leq \frac{L}{\Lambda+1},
$$

where $\Lambda$ is given by (9). Then, the boundary value problem (1) has a solution.

Proof. Consider

$$
\mathcal{C}_{L}=\left\{v \in \mathbb{N}_{o}^{p} \rightarrow \mathbb{R},\|v\| \leq L\right\}
$$

Clearly, $\mathcal{C}_{L}$ is a non-empty compact convex subset of $\mathbb{R}^{p-o+1}$. Let $T$ be a operator as defined in (18). It is clear that $T$ is a continuous operator. Therefore the main objective is to show that $T: \mathcal{C}_{L} \rightarrow \mathcal{C}_{L}$, then Theorem 8 can be invoked.

Let

$$
\Omega_{0}=\frac{L}{\Lambda+1}
$$

By using (22), (23) and Lemma 4

$$
\begin{aligned}
\|T v\| \leq & \max _{z \in \mathbb{N}_{o}^{p}}\left|A(z) g_{1}(v)+B(z) g_{2}(v)\right|+\max _{z \in \mathbb{N}_{o}^{p}} \sum_{t=o+2}^{p} G(z, t) q(t, v(t)) \\
& \leq g_{1}(v)+g_{2}(v)+\left(\frac{L}{\Lambda+1}\right) \sum_{t=0+2}^{p} \max _{z \in \mathbb{N}_{o}^{p}}[G(z, t)] \\
& \leq\left(\frac{L}{2(\Lambda+1)}\right)+\left(\frac{L}{2(\Lambda+1)}\right)+\left(\frac{L}{\Lambda+1}\right) \Lambda \\
& =\Omega_{0}(1+\Lambda)=L .
\end{aligned}
$$

Thus, $\|T v\| \leq L$ and $T: \mathcal{C}_{L} \rightarrow \mathcal{C}_{L}$. It follows at once by Brouwer fixed point theorem, that there exist a fixed point of $T$, say $v_{0} \in \mathcal{C}$, such that $\left|v_{0}(z)\right| \leq L$. The proof is complete.

Theorem 10 (See [25]). [Contraction Mapping Theorem]. Let $S$ be a closed subset of $\mathbb{R}^{n}$. Assume $T: S \rightarrow S$ is a contraction mapping, i.e., there exists a number ' $\xi$ ', $0 \leq \xi \leq 1$, such that

$$
\|T v-T u\| \leq \xi\|v-u\|,
$$

for all $v, u \in S$. Then, $T$ has a unique fixed point $v_{0} \in S$.

Theorem 11. Assume that $q(z, v), g_{1}(v)$ and $g_{2}(v)$ are Lipschitz with respect to ' $v$ ', i.e., there exists $a^{\prime}, b^{\prime}, c^{\prime}>0$, such that $\left|q\left(z, v_{1}\right)-q\left(z, v_{2}\right)\right| \leq a^{\prime}|| v_{1}-v_{2}\left\|,\left|g_{1}\left(v_{1}\right)-g_{1}\left(v_{2}\right)\right| \leq b^{\prime}|| v_{1}-v_{2}\right\|$ and $\left|g_{2}\left(v_{1}\right)-g_{2}\left(v_{2}\right)\right| \leq c^{\prime}\left\|v_{1}-v_{2}\right\|$, whenever $v_{1}, v_{2} \in C\left[\mathbb{N}_{o}^{p} \rightarrow \mathbb{R}\right]$. Then the boundary value problem (1) has a unique solution provided

$$
a^{\prime} \Lambda+b^{\prime}+c^{\prime}<1
$$

holds. 
Proof. Consider

$$
\begin{aligned}
\left\|T v_{1}-T v_{2}\right\|= & \max _{z \in \mathbb{N}_{o}^{p}}\left|T v_{1}-T v_{2}\right| \\
\leq & \max _{z \in \mathbb{N}_{o}^{p}} \sum_{t=o+2}^{p} G(z, t) q\left(t, v_{1}\right)-\max _{z \in \mathbb{N}_{o}^{p}} \sum_{t=o+2}^{p} G(z, t) f\left(t, v_{2}\right) \\
& +\max _{z \in \mathbb{N}_{o}^{p}}\left(\frac{p-z}{p-o}\right) H_{\vartheta-2}(z, \rho(o)) g_{1}\left(v_{1}\right)-\max _{z \in \mathbb{N}_{o}^{p}}\left(\frac{p-z}{p-o}\right) H_{\vartheta-2}(z, \rho(o)) g_{1}\left(v_{2}\right) \\
& +\max _{z \in \mathbb{N}_{o}^{p}} \frac{(z-o)^{\overline{\vartheta-1}}}{(p-o)^{\overline{\vartheta-1}}} g_{2}\left(v_{1}\right)-\max _{z \in \mathbb{N}_{o}^{p}} \frac{(z-o)^{\overline{\vartheta-1}}}{(p-o)^{\overline{\vartheta-1}}} g_{2}\left(v_{2}\right) \\
\leq & \sum_{t=o+2}^{p} \max _{z \in \mathbb{N}_{o}^{p}}[G(z, t)]\left|q\left(t, v_{1}\right)-q\left(t, v_{2}\right)\right| \\
& +\max _{z \in \mathbb{N}_{o}^{p}}\left[\left(\frac{p-z}{p-o}\right) H_{\vartheta-2}(z, \rho(o))\right]\left|g_{1}\left(v_{1}\right)-g_{1}\left(v_{2}\right)\right| \\
& +\max _{z \in \mathbb{N}_{o}^{p}}\left[\frac{(z-o)^{\overline{\vartheta-1}}}{(p-o)^{\bar{\vartheta}-1}}\right]\left|g_{2}\left(v_{1}\right)-g_{2}\left(v_{2}\right)\right| \\
\leq & \Lambda a^{\prime}\left\|v_{1}-v_{2}\right\|+b^{\prime}\left\|v_{1}-v_{2}\right\|+c^{\prime}\left\|v_{1}-v_{2}\right\| \\
\leq & \left(\Lambda a^{\prime}+b^{\prime}+c^{\prime}\right)\left\|v_{1}-v_{2}\right\| .
\end{aligned}
$$

Thus, using (24) $T$ is a contraction on $\mathbb{R}^{p-o+1}$. Hence, by Theorem 10, the result follows. The proof is complete.

Example 1. Suppose, $\vartheta=1.1$ and $o=0, p=10, q(z, v)=\frac{\sin (v)}{15+z}, g_{1}(v)=\frac{\sum_{t=o}^{p} v(t)}{20}$ and $g_{2}(v)=\frac{\sum_{t=o}^{p} v(t)}{10}$. Then, (1) becomes

$$
\left\{\begin{array}{l}
-\left(\nabla_{\rho(0)}^{1.1} v\right)(z)=\frac{\sin (v)}{15+z}, \quad z \in \mathbb{N}_{2}^{10}, \\
v(0)=\frac{\sum_{t=o}^{p} v(t)}{20}, \quad v(10)=\frac{\sum_{t=o}^{p} v(t)}{10} .
\end{array}\right.
$$

Clearly, $q(z, v), g_{1}(v)$ and $g_{2}(v)$ are Lipschitz with respect to $v$ with Lipschitz constant $a^{\prime}, b^{\prime}$ and $c^{\prime}$, respectively. Here $a^{\prime}=\frac{1}{15}, b^{\prime}=\frac{1}{20}$ and $c^{\prime}=\frac{1}{10}$. Then

$$
\Lambda=\left(\frac{(p-o)(\vartheta-1)+1}{\vartheta}\right)^{\overline{\vartheta-1}}\left(\frac{(p-o-1)}{\vartheta \Gamma(\vartheta+1)}\right)=8.05 .
$$

and

$$
\left(a^{\prime} \Lambda+b^{\prime}+c^{\prime}\right)=0.6866<1 .
$$

Thus, by Theorem 11 the boundary value problem (25) has a unique solution.

Example 2. Suppose, $\vartheta=1.5$ and $o=0, p=6, q(z, v)=\frac{1}{20}\left(\sqrt{v}+v^{2}\right), g_{1}(v)=\frac{1}{11} v(1)-$ $\frac{1}{23} v(4)$ and $g_{2}(v)=\frac{1}{9} v(5)-\frac{1}{10} v(2)$. Then, (1) becomes

$$
\left\{\begin{array}{l}
-\left(\nabla_{\rho(0)}^{1.5} v\right)(z)=\frac{1}{20}\left(\sqrt{v}+v^{2}\right), \quad z \in \mathbb{N}_{2}^{6}, \\
v(0)=\frac{1}{11} v(1)-\frac{1}{23} v(4), \quad v(6)=\frac{1}{9} v(5)-\frac{1}{10} v(2) .
\end{array}\right.
$$


Here for a given values of $o, p$ we get $\bar{\lambda}=0.1191$ and $\eta=0.77$. We see that $q(z, v)$ satisfies condition (H1) and (H4), also by taking $r_{2}=2$ we have that

$$
q(z, v)=\frac{1}{20}\left(\sqrt{v}+v^{2}\right) \leq \frac{1}{20}\left(\sqrt{r_{2}}+r_{2}^{2}\right)=0.270<\frac{\eta r_{2}}{2}=0.77,
$$

and $g_{1}, g_{2}$ satisfy conditions (G1) and (G2). Thus, all conditions of Theorem 6 are satisfied, hence (26) has at least two positive solutions $v_{1}$ and $v_{2}$ such that $0<\left\|v_{1}\right\|<2<\left\|v_{2}\right\|$.

\section{Conclusions}

In this paper, we constructed the Green's function corresponding to the boundary value problem (1), and stated a few of its properties. We studied the existence of at least one and at least two positive solutions for the boundary value problem (1) using Guo-Kranoselskii fixed point theorem on cones, and obtained sufficient conditions on the existence and uniqueness of solutions for the proposed class of boundary value problems using Brouwer and contraction mapping theorems, respectively. Finally, we demonstrated the applicability of established results with a few examples. To the best of our knowledge, there is no prior progress made with non-local conditions in the nabla case.

Author Contributions: All authors equally contributed this paper and approved the final version. All authors have read and agreed to the published version of the manuscript.

Funding: This research received no external funding.

Institutional Review Board Statement: Not applicable.

Informed Consent Statement: Not applicable.

Data Availability Statement: No new data were created or analyzed in this study. Data sharing is not applicable to this article.

Acknowledgments: The authors would like to express their full thanks to the respected editorial office and also author N. S. Gopal acknowledges support received from CSIR-HRDG New Delhi, Government of India.

Conflicts of Interest: We declare that no competing interest exist regarding this manuscript.

\section{References}

1. Miller, K.S.; Ross, B. Fractional difference calculus. In Proceedings of the International Symposium on Univalent Functions, Fractional Calculus, and Their Applications, Koriyama, Japan, 1-5 May 1988; pp. 139-152.

2. Kilbas, A.A.; Marichev, O.I.; Samko, S.G. Fractional Integrals and Derivatives. Theory and Applications; Translated from the 1987 Russian Origina. Revised by the authors; Gordon and Breach Science Publishers: Yverdon, Switzerland, 1993; Volume xxxvi, 976p.

3. Podlubny, I. Fractional Differential Equations. An Introduction to Fractional Derivatives, Fractional Differential Equations, to Methods of Their Solution and Some of Their Applications, 198. Mathematics in Science and Engineering; Academic Press, Inc.: San Diego, CA, USA, 1999.

4. Kilbas, A.A.; Srivastava, H.M.; Trujillo, J.J. Theory and Applications of Fractional Differential Equations. 204. North-Holland Mathematics Studies; Elsevier Science B.V.: Amsterdam, The Netherlands, 2006.

5. Gray, H.L.; Zhang, N.F. On a new definition of the fractional difference. Math. Comput. 1988, 50, 513-529. [CrossRef]

6. Miller, K.S.; Ross, B. Ellis Horwood Series: Mathematics and Its Applications; Srivastava, H.M., Owa, S., Eds.; Ellis Horwood Ltd.: Chichester, UK; Halsted Press [John Wiley and Sons, Inc.]: New York, NY, USA, 1989; 404p.

7. Atici, F.M.; Eloe, P.W. Discrete fractional calculus with the nabla operator. Electron. J. Qual. Theory Differ. Equ. 2009, I, 12.

8. Wu, F. Nabla Fractional Calculus and Its Application in Analyzing Tumor Growth of Cancer. Master's Thesis, Western Kentucky University, Bowling Green, KY, USA, 2012.

9. Huang, L; Bae, Y. Chaotic Dynamics of the Fractional-Love Model with an External Environment. Entropy 2018, 20, 53. [CrossRef] [PubMed]

10. Huang, L.; Bae, Y.-C. Non-linear Behavior in Fractional-Order Romeo and Juliet's Love Model Influenced by External Force with Fuzzy Function. Int. J. Fuzzy Syst. 2018, 21, 2018.

11. Goodrich, C.; Peterson, A.C. Discrete Fractional Calculus; Springer: Cham, Switzerland, 2015.

12. Ahrendt, K.; De Wolf, L.; Mazurowski, L.; Mitchell, K.; Rolling, T.; Veconi, D. Initial and Boundary Value Problems for the Caputo Fractional Self-Adjoint Difference Equations. Enlight. Pure Appl. Math. 2016, 2, 1. 
13. Goar, J.S. A Caputo Boundary Value Problem in Nabla Fractional Calculus. Ph.D. Thesis, The University of Nebraska, Lincoln, NE, USA, 2016; 112p.

14. Ikram, A. Lyapunov inequalities for nabla Caputo boundary value problems. J. Differ. Equ. Appl. 2019, 25, 757-775. [CrossRef]

15. Brackins, A. Boundary Value Problems of Nabla Fractional Difference Equations. Ph.D. Thesis, The University of Nebraska, Lincoln, NE, USA, 2014; 92p.

16. Gholami, Y.; Ghanbari, K. Coupled systems of fractional $\nabla$-difference boundary value problems. Differ. Equ. Appl. 2016, 8 , 459-470. [CrossRef]

17. Jonnalagadda, J.M. On two-point Riemann-Liouville type nabla fractional boundary value problems. Adv. Dyn. Syst. Appl. 2018, 13, 141-166.

18. Jonnalagadda, J.M. On a nabla fractional boundary value problem with general boundary conditions. AIMS Math. 2020, 5, 204-215. [CrossRef]

19. Jonnalagadda, J.M. Existence results for solutions of nabla fractional boundary value problems with general boundary conditions. Adv. Theory Non-Linear Anal. Appl. 2020, 4, 29-42. [CrossRef]

20. Jonnalagadda, J.M. An ordering on Green's function and a Lyapunov-type inequality for a family of nabla fractional boundary value problems. Fract. Differ. Calc. 2019, 9, 109-124. [CrossRef]

21. Jonnalagadda, J.M. Discrete fractional Lyapunov-type inequalities in nabla sense. Dyn. Contin. Discrete Impuls. Syst. Ser. A Math. Anal. 2020, 27, 397-419.

22. Jonnalagadda, J.M. Lyapunov-type inequalities for discrete Riemann-Liouville fractional boundary value problems. Int. J. Differ. Equ. 2018, 13, 85-103.

23. Goodrich, C. Existence and uniqueness of solutions to a fractional difference equation with non-local conditions. Comput. Math. Appl. 2011, 61, 191-202. [CrossRef]

24. Goodrich, C.S. On positive solutions to non-local fractional and integer-order difference equations. Appl. Anal. Discrete Math. 2011, 5, 122-132. [CrossRef]

25. Agarwal, R.P.; Meehan, M.; O'regan, D. Fixed Point Theory and Applications. 141. Cambridge Tracts in Mathematics; Cambridge University Press: Cambridge, UK, 2001. 